\title{
Arbor
}

\section{El Ejército del Aire pionero en operaciones de paz y humanitarias efectuadas por España}

\section{Pedro Bernal Gutiérrez}

Arbor CLXXI, 674 (Febrero 2002), 341-366 pp.

Este artículo hará las delicias del lector interesado en conocer a fondo cómo se han desarrollado las operaciones de paz y humanitarias en las que ha intervenido el Ejército del Aire. Podrá informarse de cómo se gestaron, quiénes las realizaron, qué dificultades encontraron, qué esfuerzos tuvieron que realizar, etc.

El objetivo de este artículo, una vez establecido que el panorama estratégico actual ha cambiado y que, salvo cambios inesperados, la nueva situación deriva hacia la realización cada vez más frecuente de operaciones de paz y humanitarias, es de resaltar la participación en dichas misiones del Ejército del Aire, como uno de los instrumentos de la acción exterior del Estado.

Da interés al artículo el hecho de que la información que se nos ofrece esté obtenida directamente de las unidades y personal que realizaron dichas misiones.

Dada la cantidad de misiones efectuadas en Europa, Asia, África y América, sólo se desarrollan con detalle las de Guinea Ecuatorial y Namibia.

Se llega a la conclusión de que, sin menosprecio de la labor realizada por otros Ejércitos u Organizaciones, el Ejército del Aire ha sido, es y seguirá siendo uno de los pilares fundamentales en que se base la actuación exterior de España en este tipo de operaciones. 


\section{Introducción}

Los grandes cambios acaecidos en la sociedad internacional durante los últimos años han conllevado un aumento de las operaciones de paz y humanitarias por parte de Naciones Unidas y organizaciones como la OTAN, la UEO o la OSCE.

España ha intensificado su participación en estas misiones y su acción exterior se ha reforzado con los éxitos obtenidos por nuestras Fuerzas Armadas, operando en condiciones distintas a las habituales.

Esas actuaciones tienen sus antecedentes en las misiones realizadas por el Ejército del Aire mediante vuelos de ayuda humanitaria, destacamento permanente en Guinea Ecuatorial o despliegue de una unidad en Namibia.

\section{España y el espíritu solidario y de cooperación internacional}

La voluntad de asumir la responsabilidad que corresponde a España, como país de creciente importancia en la comunidad de naciones, en el mantenimiento de la paz y seguridad internacionales, en el apoyo humanitario y, en general, en la cooperación internacional en apoyo de la paz y estabilidad, viene expresada en el Preámbulo de la Constitución de 1978 «colaborar en el fortalecimiento de unas relaciones pacíficas y de eficaz cooperación entre todos los pueblos de la tierra» y es tenida en cuenta en el planeamiento de la Defensa Nacional.

A lo largo de la década de los ochenta la política de seguridad española fue definiendo con pasos concretos su participación en organizaciones internacionales ${ }^{1} \mathrm{y}$, en particular, en las estructuras de paz y seguridad. La vocación de contribuir activamente a la paz mundial con recursos propios fue expresada por el Rey Don Juan Carlos ante la Asamblea General de la ONU en 1986 como también lo había hecho el presidente González en 1985.

Más recientemente, la Directiva de Defensa Nacional 1/96 afirma en su Preámbulo que España se encuentra hoy plenamente comprometida en la consecución de un orden internacional más estable y seguro, basado en la convivencia pacífica, en la defensa de los derechos humanos ${ }^{2}$ y en el respeto a las normas del derecho internacional, y que este compromiso es evidente con nuestra presencia y decidida participación en operaciones de paz.

Por último, la Directiva de Defensa Nacional $1 / 00^{3}$, en su Preámbulo, reconoce el valor de las Fuerzas Armadas como «instrumento de la acción 
El Ejército del Aire pionero en operaciones de paz...

exterior del Estado» y que «actúan, cada vez con mayor frecuencia, en tareas de ayuda humanitaria o en operaciones de gestión de crisis o de prevención de conflictos, supuestos de actuación radicalmente distintos, aunque no excluyentes, de los (ya citados) de disuasión o defensa territorial contra un ataque generalizado, únicos ejes de la estrategia occidental durante la Guerra Fría».

Como uno de los tres objetivos prioritarios subraya: «contribuir a las misiones de ayuda humanitaria y operaciones de gestión de crisis que realicen las organizaciones internacionales y europeas a las que España pertenece».

En la segunda de las ocho líneas básicas en que se concretará el desarrollo de la política de defensa establece: «complementar la acción exterior del Estado en los ámbitos de seguridad y defensa, contribuir al mantenimiento de la paz, la seguridad y estabilidad internacionales y hacer valer nuestra presencia en las organizaciones de seguridad y defensa colectivas, especialmente en lá Alianza Atlántica y en la Unión Europea.

España apoyará los procesos de implantación de las estructuras políticas y militares europeas y participará activamente en las iniciativas de control y desarme».

Es interesante resaltar que las misiones de ayuda humanitaria y las de gestión de crisis a que se alude en la Directiva no se limitan a las misiones u operaciones realizadas bajos los auspicios de ONU o de una organización determinada, sino que tienen un significado mucho más amplio.

\section{Participación en operaciones de paz y humanitarias}

Naciones Unidas es la organización internacional con mayor experiencia en el planeamiento y desarrollo de operaciones de mantenimiento de la paz. Su preeminencia en el campo de la prevención y gestión de conflictos ha sido reconocida por otras organizaciones de carácter regional que, en ocasiones, actúan asumiendo funciones delegadas de la ONU o incluso aportan capacidades de mando y control y de medios para el empleo de la fuerza.

En la Carta Fundacional de la ONU no se recoge de forma explícita la definición de las Operaciones para el Mantenimiento de la Paz (OMP), para las que no existe tampoco una doctrina ni una estructura fija, sin embargo, la necesidad de desarrollar un instrumento para poner en práctica las medidas que establece el artículo 40 de la Carta Fundacional ha 
dado lugar a llenar de forma pragmática un vacío no previsto en el momento de crear la institución.

La propia evolución de la situación internacional ha condicionado el tipo de misiones y los cometidos a desarrollar, abriendo un amplio abanico de posibilidades que van desde la aplicación de medidas preventivas a las de verificación de acuerdos, desde la presencia de observadores al empleo de la fuerza o desde la resolución de conflictos a la reconstrucción de la sociedad y el apoyo en situaciones de catástrofes. Igualmente se ha producido un proceso de cambio en cuanto a organizaciones implicadas, ya que si inicialmente solamente participaba la ONU, en las últimas operaciones se han incorporado otras organizaciones de seguridad regionales.

\subsection{Durante los años de la Guerra Fría}

Cabría distinguir una primera etapa que va desde la creación de la ONU en 1945 hasta la caída del muro de Berlín, en 1989, de poca intensidad y con misiones de paz condicionadas por la existencia de la guerra fría y la influencia de los dos grandes bloques ${ }^{4}$. Con esos obstáculos, el papel de la ONU fue limitado y su capacidad, a veces, puesta en tela de juicio, sin embargo, hay que destacar la ayuda a refugiados, su presencia en zonas de emergencia y su valor como foro internacional de discusión para evitar la escalada de los numerosos conflictos de la época. Ese papel tuvo su reconocimiento con la concesión del Premio Nobel de la Paz en 1988.

Durante ese tiempo España, que se hizo miembro de la ONU en 1955, no participó en ninguna de las trece misiones de paz de esta Organización ${ }^{5}$, sin embargo, sí llevó a cabo de forma prácticamente aislada misiones humanitarias y de cooperación internacional, mediante vuelos de ayuda para transporte de personas, alimentos, medicamentos o material de diversos tipos, con ocasión de catástrofes naturales o de situaciones de crisis. También mantuvo un destacamento permanente de dos aviones en Guinea Ecuatorial, durante quince años, como parte del programa de cooperación de España con ese país.

Para el Ejército del Aire, esa actividad pionera comenzó a adquirir un carácter de naturaleza a partir de 1960 y con sus unidades de transporte desarrolló una experiencia de colaboración con el Ministerio de Asuntos Exteriores, de disponibilidad, de rapidez de reacción y de operación continuada en toda clase de condiciones, lejos de nuestro país, consolidada ya mucho antes de que España participara de lleno en las misiones de la ONU. Como consecuencia de esa capacidad, el Estado Mayor del Aire 
El Ejército del Aire pionero en operaciones de paz...

fue consultado en la primera ocasión en que España fue invitada a participar en una misión de paz, en este caso en Namibia, en virtud de la Resolución 435/78.

\subsection{Cambios ante la nueva situación}

En una segunda etapa, a partir de 1989, y coincidiendo con el final de la guerra fría y la aparición de un nuevo escenario estratégico, se replanteó la concepción de las misiones de paz y se pasó de la ayuda para solucionar conflictos entre Estados a hacer frente con frecuencia a crisis locales, guerras civiles y desastres humanitarios. La creciente demanda de intervenciones de la ONU ${ }^{6}$ obligó incluso a crear en 1994 un Sistema de Fuerzas de Reserva.

En este periodo, España, además de continuar con las misiones humanitarias mediante vuelos de ayuda y destacamentos del Ejército del Aire, como el de un avión T-19 «CN-235» en Ruanda, comenzó una participación intensa en numerosas misiones, empleando fuerzas de los tres Ejércitos y de la Guardia Civil, de forma prolongada y en diversos escenarios, donde se han desarrollado prácticamente toda clase de los tipos de misiones recogidas en la clasificación hecha en 1992 por el entonces Secretario General de la ONU, Butros Gali, tanto las de carácter diplomático preventivo como las de establecimiento (peacemaking), mantenimiento (peacekeeping), imposición (peace enforcement) o consolidación de la paz (peace building), así como las de carácter humanitario.

Es de destacar que los esfuerzos de participación que España realizó desde un principio tuvieron su respuesta con ocasión de las operaciones que la ONU había previsto, de forma coordinada y simultánea, en el escenario del Africa Austral. La contribución española se plasmó en el envío de observadores del Ejército de Tierra a UNAVEM-I en Angola y en el despliegue de una unidad autónoma de transporte del Ejército del Aire en Namibia para la misión de UNTAG. La rapidez de reacción y la efectividad con que se llevó a cabo por primera vez el despliegue de una unidad española en una misión ONU estuvieron respaldadas por la madurez y experiencia alcanzada en años anteriores.

Para el Ejército del Aire, esta primera operación supuso recibir su «bautismo de paz» a nivel organización, ya que tuvo que poner en juego su capacidad de planeamiento, de operación y de apoyo logístico sostenido, para cubrir con unos medios limitados, de diversa procedencia y organizados como un Grupo de Fuerzas Aéreas autónomo, una superficie de $823.000 \mathrm{Km}^{2}$, a más de 7.000 kilómetros de distancia de sus bases naturales. 
Además de las misiones de carácter específico también tuvo que hacer un considerable esfuerzo para cubrir las nuevas necesidades de apoyo de las Fuerzas Armadas, que al comenzar a participar en misiones de la ONU, lejos de nuestras fronteras precisaban, cada vez más, de transporte aéreo.

Más recientemente, la participación de España en las operaciones de paz en los Balcanes, autorizada inicialmente por Acuerdo de Consejo de Ministros de 17 de julio de 1992 y que se ha prolongado durante estos últimos años a través de diversas operaciones, ha marcado para nuestras Fuerzas Armadas una prueba de fuego en cuanto a contribución a estas misiones, forma de operar y ámbito de actuación.

Además de la ONU, diversas organizaciones de seguridad regional han ido incorporándose, de una u otra forma, a esas operaciones, así la OTAN que ya había actuado en 1991 en tareas de pacificación con motivo de la guerra de Irak, recibió su prueba de fuego en 1993 al poner en marcha la operación "Deny Flight», para hacer respetar la zona de exclusión aérea decretada sobre Yugoslavia, en respuesta a la Resolución 816 del Consejo de Seguridad de la ONU. Con la puesta en marcha de la Fuerza de Implementación (IFOR) en 1995 la OTAN afrontó su primera misión terrestre y ha continuado actuando en la región mediante otras misiones de paz.

También la Organización para la Seguridad y la Cooperación en Europa (OSCE) realizó su primera misión en agosto de 1992 en Macedonia y ha enviado observadores a Croacia, además de a algunas regiones de la antigua Unión Soviética.

La Unión Europea Occidental (UEO), por su parte, participó ya en agosto de 1991 en el embargo marítimo decretado por la ONU contra Irak y tras acordar un año después, en Petersberg, las principales misiones de la UEO: las humanitarias, las de gestión de crisis y las de paz, ha actuado en Croacia, Bosnia-Herzegovina y Albania.

En lo que al Ejército del Aire se refiere, su actuación en los Balcanes y, en particular en la operación «Allied Force» en Kosovo ha supuesto participar en la primera operación de envergadura en la que la OTAN se ha visto involucrada en sus cincuenta años de existencia, haciéndolo bajo el mandato de las Naciones Unidas, en cumplimiento de las resoluciones del Consejo de Seguridad para detener el desastre humanitario en esa región ${ }^{7}$.

Hay que resaltar que para las unidades de combate que destacaron medios en la B.A. de Aviano se trató, no solamente de actuar en misiones reales de fuego, sino de hacerlo en las que presentaban mayores exigencias en cuanto a características tecnológicas de los medios a emplear y a 
El Ejército del Aire pionero en operaciones de paz...

los niveles de capacidad y adiestramiento del personal, condiciones que sólo podían cumplir muy contadas naciones, aparte de los Estados Unidos.

Los medios de apoyo al combate desarrollaron igualmente una actividad intensa y tuvieron su bautismo de fuego, cabe destacar aquí el T-12 «Aviocar» destacado en Vicenza que fue alcanzado por un misil y consiguió recuperarse gracias a la capacidad de su tripulación, las misiones de reabastecimiento en vuelo o la labor de los equipos de control aero-táctico (TACP) para la conducción de aviones aliados.

Para España, los éxitos cosechados se han convertido en un activo muy importante de su acción exterior, gracias al buen hacer de nuestros soldados, su alto grado de profesionalidad y su capacidad para actuar en conflictos prolongados lejos de nuestras fronteras, así como para trabajar coordinadamente con nuestros aliados en misiones internacionales y para colaborar con organizaciones no gubernamentales ${ }^{8}$. En la opinión pública estas circunstancias han permitido evolucionar desde un cierto recelo hacia las «aventuras militares exteriores» a una aceptación de las misiones de paz como una de las razones que justifican la existencia de las Fuerzas Armadas.

\section{Vuelos de ayuda humanitaria}

De acuerdo con la clasificación de las misiones de la ONU, realizada en 1992 por su entonces secretario general Butros Gali, la ayuda humanitaria es una medida compatible con todas las otras medidas. Fuerzas militares garantizan y protegen el reparto de ayuda humanitaria dirigido por agencias especializadas de las Naciones Unidas o por organizaciones civiles.

Las operaciones de ayuda humanitaria están dirigidas a socorrer a las víctimas de catástrofes humanas, que pueden ser causadas por la naturaleza, por conflictos armados o por determinadas situaciones de inestabilidad o crisis de origen político o social.

Esas situaciones suelen ser imprevisibles, estar afectadas por un carácter de urgencia e, incluso, tener una amplia repercusión en la región donde se producen.- Normalmente exigen una rápida capacidad de reacción y las condiciones en que se desarrollan son desfavorables y, a veces, hostiles.

Hoy en día no se limitan al reparto de alimentos y artículos de primera necesidad, a la ayuda sanitaria o a la reparación de infraestructuras, ya que es frecuente tener que reconstruir una organización social ca- 
paz de proporcionar servicios vitales de forma estable y segura. En ellas suelen participar representantes de diversas naciones y de organizaciones no gubernamentales y se plantean unas grandes necesidades de coordinación.

La capacidad de los medios aéreos para movilizarse y transportar con rapidez a lugares alejados medios o equipos de primera necesidad y para apoyar las necesidades de transporte en la zona, junto a su flexibilidad para adaptarse a distintos tipos de demanda de actuación, ha hecho de estos medios un instrumento de gran utilidad con ocasión de crisis humanitarias. Cuando esos medios tienen, además, la posibilidad de operar sin necesidad de infraestructuras o medios de apoyo complicados, las ventajas de su empleo se acrecientan notablemente.

No es de extrañar, por tanto, que aviones del Ejército del Aire hayan intervenido desde hace décadas en numerosas ocasiones y que sus unidades de transporte hayan adquirido una experiencia para operar en las condiciones citadas anteriormente que, han servido de base para participar en operaciones más complejas o de mayor entidad.

Antes de citar algunas de esas ocasiones, sería conveniente resaltar el espíritu de solidaridad y de cooperación internacional que ha animado a los aviadores españoles desde los primeros momentos en que la creciente capacidad y autonomía de sus aeronaves les permitieron alcanzar metas cada vez más alejadas. Recientemente, con ocasión de los actos en conmemoración del 75 aniversario de «los grandes vuelos», entre ellos el del «Plus Ultra» y su periplo sudamericano, se ha mencionado la importante carga de carácter diplomático que transportaron consigo aquellos pioneros de los vuelos transoceánicos, en forma de mensajes de amistad y solidaridad para con nuestros países hermanos, cuyas relaciones con la antigua metrópoli habían quedado muy deterioradas después de alcanzar la independencia.

\subsection{Las primeras experiencias}

Las unidades del Ejército del Aire, en la medida en que dispusieron de medios adecuados, comenzaron a desarrollar una amplia colaboración con los diversos ministerios y a aumentar su aparición en la escena internacional, entre otras actuaciones, mediante vuelos de socorro y ayuda a la Cruz Roja. Un caso a destacar fue el acontecido el 1 de marzo de 1960 en que se realizó un gran esfuerzo de ayuda humanitaria y socorro con ocasión del maremoto de Agadir, en Marruecos.

Ante las primeras noticias, el Gobierno español dispuso un plan urgente de ayuda y ordenó la salida esa misma noche de un avión AD.1 
El Ejército del Aire pionero en operaciones de paz...

«Grumman» del 802 Escuadrón, del Servicio de Búsqueda y Salvamento (SAR) de la Base Aérea de Gando, para hacer una evaluación de la situación y obtener fotografías de los daños a muy baja altura. De regreso a Gando el avión recogió un equipo médico, así como material sanitario y medicamentos, siendo esta la primera ayuda eficaz que llegó a Agadir. En ese mismo día todavía tuvo ocasión de evacuar 12 heridos graves a Casablanca antes de recuperarse en Gando, tras haber realizado 14:40 horas de vuelo.

Al Ala 35 de Transporte de Getafe, cuyo primer Escuadrón se formó en mayo de 1956 con aviones DC-3 y más tarde se aumentó con aviones DC-4, se le ordenó el envío en tiempo récord de cinco aviones «DC-4» y catorce $« \mathrm{DC}-3{ }^{9}$ a Marruecos, donde se concentraron con aviones de otros países. Los aviones españoles del ala 35 fueron desplegados en las bases de Rabat -Sale, Nousseaur, Port Lyautey y Casablanca- Cazes. Los «DC3» se dedicaron al transporte de tropas de Tánger a Agadir, mientras que los «DC-4» se emplearon en el transporte de material y maquinaria desde la ciudad de Uxda. El total de servicios realizados por la Unidad fue de 172, necesitándose para ello más de 300 horas de vuelo.

La actuación de las unidades del Ejército del Aire recibió el reconocimiento del Reino de Marruecos y del Gobierno español por su eficiente ayuda y el alto comportamiento humano de sus dotaciones.

También se pueden citar otras ayudas de menor entidad con motivo del terremoto en Amman en octubre de 1970 o la ayuda a los refugiados de Klar-Toum.

De las experiencias vividas en las misiones de esa época da una idea la que tuvo lugar en Guinea Ecuatorial en 1969, con ocasión de la recogida de una comisión interministerial española, en la que se confiscó el avión, se retuvo a la tripulación varias horas por soldados armados y se llegó a amenazar con fusilamientos ${ }^{10}$.

A partir de 1976, con la progresiva sustitución de sus aviones por el T-12 «Aviocar», transporte ligero de fabricación nacional, disminuyó momentáneamente la presencia internacional del Ala 35. No obstante, todavía se le siguieron asignando misiones de pequeña entidad, como es el caso de la que se llevó a cabo en Mauritania en 1988, para transportar vacunas enviadas por la Cruz Roja, personal de la Embajada y un delegado de la Cruz Roja.

El punto de destino era un lugar en el desierto, a dos horas de vuelo de Nuakchott, que no había sido visitado desde la Segunda Guerra Mundial y que no contaba con infraestructura alguna ni ayudas para la navegación. La zona de aterrizaje era la señalada por dos sábanas en el suelo y, dado lo precario del paraje, la tripulación decidió hacer la entrega 
sin parar motores. Sin embargo, ante el emocionado e insistente agradecimiento de aquellas gentes, el comandante de aeronave hubo de descender del avión para recibir un tesoro de incalculable valor, dos botellas de agua mineral.

En 1979 las especiales características del «Aviocar» y su capacidad de operar de forma muy autónoma permitieron iniciar una importante misión en Guinea, que merece mención aparte. Más tarde, en 1989, esa experiencia hizo posible la participación de la Unidad en la misión UNTAG de la ONU en Namibia, lo que también es digno de un tratamiento separado.

La llegada en 1990 del avión T-19 «CN-235» a la Unidad aumentó su capacidad para participar en misiones humanitarias de carácter internacional, complementando las que realizaba el «Hércules» $\mathrm{y}$, aprovechando las características de operación autónoma, para realizar un destacamento de un avión y 19 personas en Ruanda desde el 19 de septiembre de 1994 al 24 de marzo de 1995, en apoyo de la misión UNAMIR con ocasión de los graves incidentes de origen racial que tuvieron lugar en esa región del Africa Central. En total, se hicieron seis relevos del personal, se volaron 403 horas y se transportaron 2.461 pasajeros y 48 toneladas de carga. Todo ello con una infraestructura mínima y en unas condiciones meteorológicas y ambientales que llegaban a poner al personal al límite de sus capacidades.

\subsection{Una actividad que se hace habitual}

En abril de 1973, con la llegada del avión Hércules C-130 a Zaragoza y la creación del 301 Escuadrón, que en septiembre de 1978 se transformó en Ala 31, dio comienzo una etapa de intensa actividad internacional ${ }^{11}$. El nuevo avión, con una carga máxima cercana a las 17 toneladas, una velocidad de crucero de $540 \mathrm{Km}$./hora, un alcance máximo superior a 16.000 kilómetros, diseñado para operar en campos de aterrizaje mínimamente preparados y de reducidas dimensiones y dotado con aviónica y equipos que le permiten operar en toda clase de condiciones atmosféricas y en territorios con escasas ayudas a la navegación, ofrecía unas posibilidades idóneas para las características de las misiones humanitarias.

En esas circunstancias y ante la efectividad con que se llevaron a cabo cuantas misiones le fueron encomendadas, esas actividades se convirtieron en habituales, superando al número del resto de misiones, y entre las tripulaciones se estableció prácticamente un turno rotatorio de alarma. En ocasiones la unidad llegó a destacar todos sus efectivos fuera de España para hacer frente a emergencias y misiones de ayuda humanitaria, 
El Ejército del Aire pionero en operaciones de paz...

convirtiéndose en su caballo de batalla, siempre dispuesto a reaccionar en cuestión de horas en cuantas ocasiones se presentaban para demostrar el espíritu solidario y de cooperación internacional de España.

La primera misión de ayuda humanitaria de la Unidad se realizó en febrero de 1975 y consistió en un cargamento de alimentos y medicinas a la zona de Malí y Níger para paliar los efectos de la hambruna ${ }^{12}$. Con esta misión se iniciaba una serie continuada de actuaciones que en la actualidad es del orden de varios centenares, algunas de las cuales se citan a continuación.

El bautismo de fuego en la ejecución de operaciones sobre territorio hostil lo recibió la Unidad en 1979, con ocasión de la evacuación de la colonia española y de otros países de Nicaragua, como consecuencia de la guerra civil en este país. La operación constó de tres expediciones ${ }^{13}$.

La primera tuvo lugar entre el 11 y el 20 de junio de 1979 y consistió en un T- 10 que partió de España a petición del Ministerio de Asuntos Exteriores para evacuar de la capital Managua a ciudadanos españoles en peligro. Tras vencer diversas dificultades de tipo diplomático y otras de carácter técnico y operativo debidas a averías, carencia de radioayudas, de cartografía de la zona y de información adecuada, el aterrizaje se efectuó mediante una maniobra complicada para evitar el fuego cruzado en la ciudad y el ametrallamiento que ya se había realizado sobre otro C130 colombiano que le había precedido. En ese ambiente se evacuaron a Costa Rica, mediante once vuelos, a 1.500 personas (de ellos 460 españoles) y en los viajes de regreso se transportaron a Nicaragua 24 toneladas de material de la Cruz Roja. Si durante los tres primeros días se evacuaron sólo españoles, con la colaboración directa del Embajador para asegurar una organización fluida, después se continuó con costarricenses y mejicanos. En total se volaron 62 horas y las tripulaciones (10 hombres) tuvieron que mantener un servicio permanente de alarma.

$\mathrm{Al}$ no conseguir la Cruz Roja fletar aviones para volar en la zona, por los riesgos existentes, se organizó del 2 al 13 de julio una segunda expedición, con el mismo avión pero esta vez con experiencia de cómo operar allí. Con él se organizó un puente aéreo entre San José, Managua y Guatemala y con un total de ocho vuelos y 49 horas de vuelo se evacuaron 740 personas de diferentes países y se transportaron de regreso 80 toneladas de material sanitario y productos alimenticios.

Entre el 8 y el 17 de agosto se organizó una tercera expedición con dos aviones (un T-10 y un TK-10) con configuración mixta de asientos y camillas, para trasladar un hospital de campaña de 26 toneladas y personal sanitario del Ejército de Tierra hasta un total de 34 pasajeros. Esta vez las dificultades tuvieron su origen en los problemas de carga y esti- 


\section{Pedro Bernal Gutiérrez}

ba de un equipo complejo y pesado. En total se volaron 85 horas entre los dos aviones.

Estas expediciones, y en particular la primera, tuvieron una gran repercusión en los medios de comunicación en Centroamérica y en España, recogiéndose comentarios muy elogiosos sobre su efectividad, brillantez y generosidad, entre otros.

Otra situación de riesgo fue la vivida el 31 de diciembre de 1978, durante la guerra civil en el Líbano, con ocasión del transporte del Presidente de la Cruz Roja y diez toneladas de material a Beirut.

Desde 1979 a 1994, dentro del programa de cooperación con Guinea Ecuatorial, en forma de transporte de ayuda humanitaria o de apoyo a la misión española, incluido el destacamento de los dos T-12, se realizaron 54 misiones de T-10 «Hércules» (con 1.535 horas de vuelo, 11.252 pasajeros y 1.702 toneladas transportadas). La carencia de medios de apoyo en estas misiones hacía que cualquier incidencia se transformase en un problema de difícil resolución, como ocurrió en uno de los primeros vuelos, en que para cambiar un motor hubo que recurrir a una grúa de las empleadas por los madereros e improvisar un andamio con material diverso encontrado sobre la marcha.

Dentro del mismo programa se incorporó a partir de 1991 el avión T19 «CN-235» del Ala 35, el cual realizó 22 misiones (con 783 horas de vuelo, 940 pasajeros y 229 toneladas de carga transportadas).

El caso de catástrofes naturales se presentaba con frecuencia. Un ejemplo de misión para estas situaciones fue la que se organizó con motivo del temblor de tierra que alcanzó los 7 grados en la escala de Mercalli y que destruyó varias ciudades de Yugoslavia el 15 de abril de 1979. Tras vencer algunas reticencias del gobierno yugoslavo, que inicialmente trató de valerse por si mismo, se envió un avión el 24 de abril de 1979. Junto a la ayuda humanitaria se trasladaron el Presidente de la Cruz Roja y un representante del Ministerio de Asuntos Exteriores, además de un grupo de periodistas.

Otro ejemplo se produjo el 14 de octubre de1980, en respuesta al terremoto que alcanzó 7,5 grados en la escala de Richter y que había asolado la ciudad de $\mathrm{Al}$ Asnam al Oeste de Argel cuatro días antes, salió de Zaragoza el primero de un total de seis aviones, que transportaron 44 toneladas de mantas, tiendas de campaña, plasma sanguíneo, vendas y objetos de primera necesidad. Esta ayuda fue coordinada por la Cruz Roja.

Otras ocasiones que se pueden mencionar, sin pretender ser exhaustivo, son:

El terremoto en el sur de Italia en noviembre de 1980, donde un T-10 transportó 12 toneladas de mantas y medicamentos, además de al Director General de Protección Civil y al Director General de la Cruz Roja. 
El Ejército del Aire pionero en operaciones de paz...

Transporte de 13 toneladas de ayuda a la ciudad de Erzurum (Turquía), con motivo de un terremoto que causó 2.000 víctimas mortales en octubre de 1983.

Apoyo a las tareas de socorro emprendidas tras las inundaciones que sufrió la ciudad de Atar en Argelia en 1983.

Transporte de material de ayuda a Santiago de Chile con ocasión del terremoto que destruyó gran parte de la ciudad en 1985.

Cabe citar la rápida reacción ante el terremoto que se produjo en septiembre de 1985 en Ciudad de Méjico, que permitió disponer en cuestión de unas horas de un avión para transportar doce toneladas de medicinas, tiendas de campaña y camas plegables, el cual fue seguido de un segundo avión con el material del equipo de bomberos de Barcelona, especializado en ese tipo de situaciones. Igual rapidez de reacción se produjo en noviembre de 1985 para enviar doce toneladas de material sanitario y grupos electrógenos, con motivo de la erupción del volcán Arenas del Nevado del Ruiz (Colombia).

Transporte de ayuda de la Cruz Roja hasta Yaoundé, en Camerún, donde una explosión de gas en el lago Nios, en agosto de 1986, había causado la pérdida de 1.500 vidas humanas.

Participación de un avión del Ala 31 en el puente aéreo entre Dakar y Nuakchott para el traslado de cientos de refugiados, en mayo de 1989, con motivo del enfrentamiento entre Mauritania y Senegal por un conflicto de fronteras.

Transporte de una carga de ayuda humanitaria a El Salvador e intento de repatriar los cadáveres de varios religiosos españoles que habían sido asesinados, en noviembre de 1989.

Transporte de un hospital militar de campaña para auxiliar a las víctimas de un devastador terremoto en Irán en junio de 1990. En esta ocasión coordinó la Media Luna Roja y hubo que hacer frente a diversas limitaciones y condicionantes de carácter religioso.

A partir de 1989, al comenzar España a participar con sus Fuerzas Armadas en las Operaciones de Paz de la ONU, el Ejército del Aire, además de los vuelos de ayuda que había venido efectuando hasta entonces, hubo de apoyar con sus aviones a los contingentes españoles de los tres ejércitos que participaban en esas misiones, ya en pleno proceso de aumento. Tal es el caso del apoyo a la misión UNTAG entre 1989 y 1990 , mediante vuelos regulares (cada tres semanas) transportando personal de relevos, repuestos, medicinas, correspondencia y carga diversa para el destacamento del Ejército del Aire en Namibia, vuelos que también se aprovechaban para apoyar a los aviones destacados en Guinea Ecuatorial y al personal español de la misión UNAVEM en Angola. Esos 


\section{Pedro Bernal Gutiérrez}

apoyos supusieron 700 horas de vuelo y el transporte de 240 pasajeros y 231.780 kilos de carga.

Todo este esfuerzo del Ala 31, que se ha continuado ininterrumpidamente en las numerosas misiones de los noventa, mereció la imposición al estandarte de la Unidad de la Corbata al Mérito Civil en mayo de 1993.

\subsection{Creciente implicación de diversas unidades y organismos}

A las tareas de cooperación internacional fueron sumándose otras unidades del Ejército del Aire, en la medida que lo permitían sus medios, así ocurrió con el $\mathbf{4 5}$ Grupo $^{14}$ que tuvo ocasión de llevar a cabo algunos de esos vuelos ${ }^{15}$, apoyándose en la amplia autonomía, rapidez y capacidad de navegación autónoma y operación bajo cualquier condición meteorológica de sus aviones, pero con las limitaciones que impone la necesidad de pistas preparadas. Hay que tener en cuenta que el «Boeing 707» ofrece su capacidad para transportar 82 ó 134 pasajeros y sus bodegas pueden llevar hasta $10.206 \mathrm{~kg}$. En el caso del «Falcon 900», su mayor utilidad ha sido en casos de aeroevacuaciones médicas urgentes.

Se puede considerar que su primera misión de este tipo se realizó el 14 de julio de 1979 con un vuelo Madrid - Luanda - Monrovia - Ginebra - Madrid, trasladando al entonces Secretario General de Naciones Unidas. Normalmente, la mayor parte de los vuelos han sido de transporte de autoridades o de apoyo a destacamentos como los de Guinea Ecuatorial, Namibia o de la ONU en general, aunque también han actuado con ocasión de hambrunas, terremotos u otras catástrofes, como ha sido el caso de El Salvador, Colombia, Méjico o Mozambique.

En el caso de apoyo al Destacamento del Ala 35 para la Cooperación con Guinea Ecuatorial se efectuaron un total de 38 misiones entre los años 1979 y 1983. En ellas se invirtieron 471 horas de vuelo y se transportaron 4.301 pasajeros. Para el apoyo a Namibia se realizaron dos misiones, en 1989 y en 1990, con un total de 46 horas de vuelo y 617 pasajeros.

En el marco de la experiencia desarrollada en cuanto a cooperación internacional en tareas humanitarias, cabría mencionar la labor realizada por el Servicio de Búsqueda y Salvamento aéreo SAR, creado el 17 de junio de 1955 para asumir las responsabilidades adquiridas por España en ese tipo de actuaciones, en su área de responsabilidad, así como también la de cooperar con otros organismos civiles y militares en ocasión de catástrofes y calamidades públicas.

Desde el principio se establecieron acuerdos con los servicios análogos de Francia e Italia para colaborar en el Mediterráneo Occidental. Los 
El Ejército del Aire pionero en operaciones de paz...

servicios prestados no se limitaron a los accidentes aéreos, sino que abarcaron búsquedas de pesqueros y diversos tipos de barcos, evacuación de heridos y enfermos e, incluso, búsqueda y rescate de personas en el desierto del Sahara o colaboraciones en áreas marítimas muy alejadas de nuestra área de responsabilidad ${ }^{16}$.

Tras la experiencia adquirida durante esos primeros años se estableció el Convenio de cooperación entre la República de Senegal y España, firmado en Dakar en 1968 para atender las zonas de responsabilidad correspondientes a los centros de búsqueda y salvamento de Dakar y Canarias. España se responsabilizaba de actuar con sus medios SAR desplegados en Canarias y el Senegal con una aeronave de largo radio de acción y tripulación puesta a su disposición por Francia ${ }^{17}$.

Durante aquellos años en que la ONU apenas desarrollaba misiones de paz y de ayuda humanitaria, en las que, por otra parte, España no participaba con medios propios, hay que resaltar en relación con la actividad desplegada por el Ejército del Aire, que en muchos de esos casos, realizados de forma aislada o a iniciativa del Gobierno español ante catástrofes naturales o crisis humanitarias ${ }^{18}$, se trataba de afrontar en cada ocasión situaciones nuevas, no exentas de riesgos ni de incertidumbres, en las que había que sacar el máximo rendimiento a unos medios con frecuencia escasos. Todo ello supuso no solamente la adquisición de una experiencia de gran valor por parte de un gran número de tripulaciones, sino también el desarrollar y consolidar durante décadas una capacidad específica a un nivel más alto de la organización, que afectaba a diversos organismos encargados de preparar y apoyar esas misiones.

Como ejemplo de la actividad que se generaba en otros organismos del Ejército del Aire cabe citar el apoyo mediante cartografía e información aeronáutica por parte del Centro Cartográfico y Fotográfico (CECAF), el establecimiento de sistemas de comunicaciones para mantener enlace con los aviones en los lugares más distantes o el esfuerzo logístico para apoyar esa actividad y el alto nivel de disponibilidad requerido, reajustando incluso la programación de actividades de mantenimiento de los medios que quedaban operando en territorio nacional.

La capacidad para coordinar, a través del Ministerio de Asuntos Exteriores, con organismos internacionales las autorizaciones de sobrevuelo o los diversos tipos de permiso requeridos para el personal tuvo que ajustarse a las exigencias de agilidad y flexibilidad.

Tampoco hay que olvidar el aspecto sanitario, que afectaba a las necesidades de tripulaciones y destacamentos en climas y condiciones muy diferentes a la habituales y con los riesgos de enfermedades contagiosas y de carácter sanitario en general, propios de las zonas en que se opera- 
ba, por otra parte las aeroevacuaciones también planteaban su problemática particular. Esas experiencias han servido de base, por ejemplo, a dos Unidades médicas del Ejército del Aire, que fueron creadas para cubrir las necesidades que se plantean hoy en día en los nuevos escenarios: la UMAD (Unidad Médica de Apoyo al Despliegue) y la UAER (Unidad de Aeroevacuaciones).

Se puede concluir que no sólo las tripulaciones, sino las Unidades y el propio Ejército del Aire como organización, desarrollaron en esa época una labor pionera en cuanto a misiones humanitarias, si bien éstas solían ser de corta duración, tener un carácter aislado o no estaban amparadas por organizaciones internacionales en el sentido en que se concibe en nuestros días.

\section{Destacamento en Guinea Ecuatorial}

En 1979, en respuesta a la petición verbal de Obiang Nguema, que acababa de asumir la presidencia del país, España reinició su programa de cooperación económica y política con Guinea Ecuatorial en el marco de un Acuerdo de Cooperación, en cuyo Protocolo de Asistencia en materia técnica de Defensa y Seguridad, firmado en $1980^{19}$, se concretaban las tareas en materia de Defensa.

Esas tareas abarcaban cuatro áreas diferenciadas: asesoramiento al presidente y a las autoridades de Defensa; formación de los cuadros de mando e instrucción de unidades; programas eventuales de material y equipo; y apoyo logístico en el ámbito civil y militar.

En virtud de ese acuerdo se envió un equipo de asesores militares para la reorganización de las Fuerzas Armadas ecuato-guineanas y se estableció un programa de becas para la formación y especialización de personal militar de ese país

En paralelo con las actividades de carácter militar del programa de cooperación y ante las dificultades económicas por las que atravesaba Guinea Ecuatorial, se estableció un destacamento permanente, con base en la isla de Bioco, de dos aviones T-12 «Aviocar» del Ala 35 del Ejército del Aire ${ }^{20}$. Inicialmente, las penurias de alojamiento hubieron de resolverse mediante el barco «Villa de Bilbao», que sirvió de improvisado hotel a los primeros cooperantes y a las tripulaciones. El Comandante del destacamento dependía funcionalmente del Embajador español y orgánicamente del Coronel Jefe del Ala 35 pero, dadas las circunstancias, que con frecuencia dificultaban cualquier tipo de consulta, se veía obligado a ejercer un elevado nivel de autonomía en sus decisiones. 
El Ejército del Aire pionero en operaciones de paz...

A partir de junio de 1991, tras pasar el T-12 «Aviocar» al Ala 37 de Valladolid, la responsabilidad del destacamento recayó en esta Unidad, la cual contó inicialmente con el apoyo de personal del Ala 35 hasta enero de1992 y prosiguió con su propio personal hasta su finalización el 13 de marzo de 1994.

Adicionalmente y completando el programa de cooperación mediante el transporte de ayuda humanitaria a Guinea y en apoyo al propio destacamento, en forma de enlace con España, se realizaron 54 misiones de T-10 «Hércules» y, en los últimos cuatro años, 22 misiones de T-19 «CN$235 »$.

El destacamento se componía de tripulaciones, equipo de mantenimiento y una Sección de Apoyo al Transporte Aéreo (SATA) y contaba con dos comandantes pilotos, dos capitanes pilotos, tres brigadas mecánicos de mantenimiento, un brigada mecánico de electrónica y un cabo primero mecánico de mantenimiento.

Periódicamente se efectuaba una rotación de los aviones de acuerdo con el programa de inspecciones de mantenimiento establecido y se aprovechaban también los vuelos semanales de Iberia para efectuar relevos de personal y para el envío de repuestos o para enviar a España material averiado reparable o las preceptivas muestras de aceite.

Desde la capital Malabo se realizaban vuelos semanales hasta Bata y Mongomo, en la zona continental, y también mensualmente hasta la isla de Annobón, además, se realizaban vuelos no periódicos para peticiones concretas de transporte aéreo, autorizadas por la Embajada de España, como eran los traslados de autoridades o las evacuaciones médicas, que solían tener el carácter de urgentes.

En definitiva los dos aviones representaban la posibilidad de enlazar una población dispersa por imperativos geográficos. Hay que tener en cuenta que los veintiocho mil kilómetros cuadrados de su superficie están repartidos fundamentalmente entre la isla de Bioco y el continente, separados entre sí por 200 kilómetros, además de la isla de Annobón, situada a 670 kilómetros al sur de Malabo y cuyo único nexo de unión con el resto del país lo constituían estos vuelos, que habían de utilizar una pista inclinada de tierra de tan sólo setecientos cincuenta metros de longitud y ningún tipo de infraestructura. También hay pequeñas islas como Elobey y Corisco, a las que no se tenía acceso con el T-12. A partir del hundimiento del único barco guineano el «Acacio Mañé», los aviones del destacamento se convirtieron también en el único medio de enlace con el continente.

Las principales ventajas que ofrecían los aviones T-12 eran su capacidad para operar en campos cortos, poco preparados, y sus reducidas necesidades de mantenimiento y de apoyo. Por otra parte, lo ajustado de su 
autonomía y su carga, obligaban a volar al límite de autonomía para aprovechar el máximo de carga útil, circunstancia muy importante dado que se transportaban materias vitales como medicinas y víveres. En el caso de Annobón se llegó a dar combustible de los propios aviones para que se pudieran poner en marcha los dos grupos electrógenos, que constituían la única fuente de electricidad en toda la isla.

La autonomía y la velocidad de estos aviones hacían que en los traslados a o desde Guinea se realizaran unas 27 horas de vuelo y se necesitasen cinco días de viaje para recorrer 6.995 kilómetros y sobrevolar 14 países $^{21}$. El itinerario tipo por etapas para la ida era el siguiente:

Getafe-Jerez-Gando.

Gando-Nouakchott (Mauritania)-Dakar (Senegal).

Dakar-Monrovia (Liberia).

Monrovia-Abidjan (Costa de Marfil)-Lagos (Nigeria).

Lagos-Malabo.

Durante los cerca de 15 años los aviones T-12 destacados efectuaron 7.155 salidas, con un total de 12.962 horas de vuelo, que se realizaron sin ayudas a la navegación, con meteorología desfavorable, desde pistas en muy malas condiciones para las maniobras de aterrizaje y despegue, con equipos de apoyo escasos y unas condiciones de trabajo que exigieron de los equipos de mantenimiento un derroche de profesionalidad, espíritu de sacrificio e imaginación. En total se transportaron 1.714 toneladas de carga y 92.232 pasajeros, cuyo control de embarque se libró del caos gracias al esfuerzo de los miembros de la Policía Nacional del equipo de cooperación.

El Ejército del Aire también pagó un precio en vidas humanas con motivo del accidente aéreo ocurrido el día 2 de enero de 1987 en Bata, en el que fallecieron los Capitanes Salcedo y Castro y el Subteniente Evaristo, junto con el personal civil que transportaban.

Más de 700 pilotos, personal de mantenimiento y personal de apoyo pasaron por el destacamento, en turnos de mes y medio que a veces se alargaban hasta cuatro meses. Las condiciones de vida del pequeño grupo que convivía en el destacamento eran difíciles, por la escasez de medios y de infraestructura, precariedad del alojamiento, la situación sanitaria, las condiciones alimentarias y la climatología de la zona, a lo que se unía el alejamiento del entorno familiar por largos periodos, que se repetían prácticamente al ritmo de una vez al año.

El apartado sanitario merece especial mención, pues durante los primeros años del destacamento la insuficiente experiencia en medicina tropical, unida a las deficientes condiciones sanitarias de Guinea, produjeron entre el personal una incidencia de enfermedades tropicales (fundamentalmente paludismo y filaria) superior al 50\%. La experiencia 
El Ejército del Aire pionero en operaciones de paz...

adquirida con el tiempo permitió reducir esa incidencia, no obstante se puede afirmar que el $25 \%$ del personal se vio afectado por alguna de las enfermedades tropicales, cuyas secuelas se sufrieron durante años.

Del significado de la misión se puede tener una idea al recordar las palabras del párroco de Malabo, durante la misa celebrada con motivo del décimo aniversario de la presencia de los Aviocar en Guinea, en las que recordó la ayuda de los pilotos españoles para un «país pobre y necesitado que agradece a Dios los servicios de los Aviocar y la vida y el sacrificio de todos los que se desgastan para que vuelen».

En reconocimiento a la labor realizada en Guinea, el Ministro de Defensa impuso el 23 de noviembre de 1981 la Corbata de la Orden al Mérito Civil al estandarte del Ala 35.

Además de su contribución a la acción exterior y a la labor humanitaria realizada por la misión española, el destacamento de Guinea significó un paso más en el proceso de adquisición de experiencia y de capacitación que se estaba desarrollando a través de los numerosos vuelos de ayuda humanitaria. Si en una primera etapa se trataba de ir y volver, cada vez más lejos y a los lugares más difíciles, más tarde, de ir, permanecer un corto espacio de tiempo y volver e, incluso hacerlo repetidas veces, ahora se establecía por primera vez un destacamento permanente lejos de España y se operaba con la misma efectividad que si se hiciera desde "casa», haciendo un esfuerzo de adaptación a condiciones difíciles y enfrentando situaciones que pasaban de lo pintoresco a lo desesperante o increíble.

$\mathrm{Si}$ a lo anterior se añade que el destacamento formaba parte de un equipo de cooperantes que desarrollaba coordinadamente las diferentes actividades de un programa de carácter nacional, se comprueba que se estaban sentando algunas de las bases conceptuales de lo que años más tarde serían participaciones en misiones de paz de la ONU. En este sentido, cabe recordar las palabras pronunciadas en la clausura del V seminario de la «Cátedra Alfredo Kindelán» celebrada en la Escuela Superior del Aire el 23 de octubre de 1995 sobre el tema «el empleo del Poder Aéreo de Europa Occidental en Operaciones de Mantenimiento de la Paz»: para las Operaciones de $\mathrm{Paz}$ «se precisa de unidades pequeñas, autosuficientes, integradas y con una capacidad modular».

\section{Misión UNTAG en Namibia}

\subsection{Antecedentes}

El Grupo de Ayuda para la Transición en Namibia (UNTAG) fue creado inicialmente mediante la Resolución 435/78 aprobada por el Consejo 
de Seguridad el 29 de septiembre. En ella se establecía un plan para la independencia del territorio, que incluía la celebración de elecciones generales, la elección democrática de un presidente y la redacción de una Constitución ${ }^{23}$. Las dificultades que surgieron entre las partes implicadas retrasaron el comienzo de la operación, obligando a una nueva Resolución la 632/89. Con ella se cerraba un proceso de casi veinticinco años de gestiones diplomáticas y diez de preparación ${ }^{24}$.

El antecedente de esta misión lo encontramos en la consulta que hizo a principios de los ochenta el recién nombrado Secretario General de Naciones Unidas, Javier Pérez de Cuéllar, al entonces presidente del Gobierno español, Leopoldo Calvo Sotelo, sobre la disposición de España a una eventual contribución militar en una operación de supervisión de la independencia de Namibia, en el marco de la ya citada Resolución 435/78 del Consejo de Seguridad ${ }^{25}$.

La petición inicial comprendía diez aviones de transporte de despegue y aterrizaje corto y las correspondientes dotaciones de personal ${ }^{26}$. Por parte del Ministerio de Asuntos Exteriores y el Ministerio de Defensa se realizaron reuniones de estudio y coordinación de los diferentes aspectos de la participación y en el verano de 1982 se dio una respuesta afirmativa por el titular de Exteriores José Pedro Pérez Llorca. En noviembre de ese año se desplazó a la sede de la ONU una comisión militar española para tratar aspectos técnicos de la misión.

El Estado Mayor del Aire, junto con representantes del Mando Aéreo de Transporte (MATRA) y del Ala 35, llevó a cabo el planeamiento operativo de la misión, basado en el Aviocar de fabricación nacional, aprovechando la rica experiencia obtenida en el destacamento de Guinea, en el que se llevaba operando de forma continuada ya varios años.

La puesta en práctica de la operación hubo de aplazarse al condicionar Sudáfrica cualquier avance a la salida de las tropas cubanas de Angola y transcurrieron seis años hasta que la coyuntura internacional permitió que se llegara a un acuerdo entre Angola, Cuba y Sudáfrica el 22 de diciembre de 1988 y que se reanudaran los estudios de la operación, que en esta ocasión estuvo ligada a la misión de UNAVEM para preparar la retirada de las tropas cubanas de Angola en julio de 1989.

\subsection{Desarrollo de la misión}

Para cumplir con el mandato de «asegurar la rápida independencia de Namibia a través de elecciones libres e imparciales bajo el control y supervisión de las Naciones Unidas» se realizó el despliegue de representantes de 50 países, que llegaron a ser 100 durante el periodo de eleccio- 
El Ejército del Aire pionero en operaciones de paz...

nes, unos 4500 soldados y 1500 policías que operaron desde el 1 de abril de 1989 hasta marzo de 1990.

España aportó ocho aviones C-212 Aviocar del Ala 35 (Getafe), los cuales tuvieron que ser preparados y equipados en un tiempo muy bre$\mathrm{ve}^{27}$, y en la última fase se añadió un C-130 Hércules del Ala 31 (Zaragoza). La ruta a Namibia, con un recorrido de 7.847 kilómetros, que requerían casi 40 horas de vuelo para los aviones T-12, constaba de las ya conocidas etapas hasta Malabo y continuaba con:

Malabo-Libreville (Gabón).

Libreville-Luanda (Angola).

Luanda-Ondangwa (Namibia).

Ondangwa-Windhoek.

En torno a un núcleo de personal del Ala 35 se organizó una unidad de entidad Grupo independiente, con 81 personas repartidas entre la base principal en la capital Windhoek y dos pequeños destacamentos al Norte, en las importantes bases aéreas sudafricanas de Rundu y Ondangwa, en la que habría de ser la primera unidad autónoma española desplegada al servicio de la ONU. En el cuartel general de UNTAG se integraron cuatro oficiales. A través de las sucesivas rotaciones de tres meses de duración pasaron por Namibia cerca de 250 militares del Ejército del Aire.

Italia completaba el componente aéreo con ocho helicópteros y un contingente de más de doscientas personas, desplegados también en Rundu y Ondangwa en el Norte, dando cobertura a la zona más densamente poblada del país. Representantes italianos se integraron igualmente en el Cuartel General.

La misión del contingente español fue la de realizar todo tipo de apoyo por medio del transporte aéreo, en particular:

- Despliegue y redespliegue de observadores y fuerzas militares.

- Traslado de personal encargado de la ayuda técnica para la organización y desarrollo del escrutinio y verificación de resultados.

- Asistencia para la repatriación de guerrilleros del SWAPO desde Angola.

- Transporte logístico y correo.

- Ayuda médica de urgencia y aeroevacuaciones médicas.

También se realizaron diversas misiones de reconocimiento aéreo visual sobre lugares de difícil acceso para control de movimientos, concretamente sobre el río Orange en la frontera con Sudáfrica.

En el Estado Mayor de UNTAG los cometidos eran los correspondientes a planeamiento, coordinación y control de las actividades aéreas de los aviones españoles, los helicópteros italianos y medios aéreos alquilados ocasionalmente por la ONU. 
Durante el destacamento los «Aviocares» realizaron 921 misiones, totalizando cerca de 3.480 horas de vuelo y transportaron 11.881 pasajeros (40 de ellos heridos) y 414 toneladas de carga. En términos de referencia equivaldría a transportar a todo el contingente de la ONU, con su equipo, de Norte a Sur de Namibia y regreso.

Por su parte, el «Hércules» enviado para reforzar la capacidad de transporte durante los meses clave de noviembre y parte de diciembre de 1989, realizó 24 misiones y 93 horas de vuelo, transportando 1.809 pasajeros y 102 toneladas de carga.

La actuación del contingente español mereció los elogios del Secretario General de las Naciones Unidas en carta dirigida al Ministro de Asuntos Exteriores de España el 21 de marzo de 1990 y la concesión de la Medalla de la ONU a los miembros del destacamento. Por otra parte, a petición del recién estrenado Gobierno, como reconocimiento a la labor realizada, el 22 de marzo de 1990 participaron en el desfile de la independencia los cuatro últimos aviones españoles.

Finalizada la misión, el 6 de abril de 1990 el Ministro de Defensa impuso al estandarte del Ala 35 la Corbata de la Orden de Isabel la Católica

\subsection{Aspectos particulares de la misión}

La necesidad de desplegar por primera vez una unidad al servicio de la ONU obligó a definir un módulo autosuficiente, integrable operativamente en una organización relativamente compleja. Para facilitar el apoyo desde España y evitar sobrecargar a otras unidades, se le dotó con el personal y los servicios mínimos imprescindibles para llevar a cabo la misión, empleando un núcleo del Ala 35 reforzado por personal de diversos organismos y se estableció un sistema rotatorio de tres meses. Una estafeta con avión "Hércules» cada tres semanas aseguraba el transporte de repuestos, suministros y rotaciones de personal.

En el destacamento principal, en la capital, desplegaron seis aviones y la mayoría del personal. Allí se centralizaron el mantenimiento y los servicios, estableciendo una rotación de dos semanas para los dos destacamentos del norte, con un avión cada uno, lo que permitió optimizar los recursos y constituyó una de las bases del éxito de la operación.

Las comunicaciones con España estuvieron aseguradas mediante enlace radio $\mathrm{HF}$ y dado que los aviones volaban la mayor parte del tiempo sobre zonas desérticas o muy poco pobladas, también se reforzó este mismo enlace con los aviones en vuelo. El concepto de dirección centralizada se fue imponiendo progresivamente, llegándose a establecer un 
El Ejército del Aire pionero en operaciones de paz...

pequeño centro de alerta y coordinación activado la mayor parte del tiempo. Permanentemente se mantuvieron alertadas cuatro tripulaciones, especialmente durante los meses de noviembre y diciembre de 1989. En muchas ocasiones hubo que estar en el aire en menos de media hora.

Las características del avión permitieron operar en todos los rincones del país, con pistas o sin ellas, y la capacidad del reducido equipo de mantenimiento permitió que la disponibilidad media fuera superior al $90 \%$. La efectividad en la realización de misiones fue del $100 \%$.

En la sensible región de Owamboland, en el norte del país, la existencia de misiles SAM-7 en manos de grupos incontrolados, que ya habían derribado un helicóptero sudafricano, obligó a volar a 15 metros de altura, con el riesgo de choque con aves y dificultad en las maniobras de aterrizaje y despegue. Precisamente la colisión con la rama de un árbol supuso el cambio de un plano completo de un avión. Esa restricción de altura de vuelo sólo fue levantada en el mes de diciembre de 1989, en que disminuyó el nivel de tensión ${ }^{28}$.

La meteorología de la zona, correspondiente a una zona desértica, aliviada por la gran elevación del interior del país y los tres meses de la época de lluvias, entre diciembre y marzo, creó un grave problema en cuanto a potencia de los motores, resuelto cuando se descubrió la fina capa de polvo sedimentada en las celdillas de los radiadores. Más complicado fue volar en la época de lluvias, sin ayudas a la navegación y con los equipos eléctricos afectados por las situaciones tormentosas.

Las instalaciones e infraestructura de apoyo y las ayudas a la navegación, que ya habían sido relativamente insuficientes al comienzo de la misión, sufrieron un grave deterioro a medida que se retiraron las fuerzas sudafricanas y en los aeródromos vacíos se tuvo que recurrir al uso de equipos autónomos, alimentados por placas solares, para puesta en marcha de los aviones, y de balizas alimentadas por baterías para iluminación de pistas en Rundu, Ondangwa, Mpacha y Grootfontein en el Norte y Keetmanshoop en el sur. Los servicios de control de tráfico aéreo quedaron reducidos a la capital y en resto del país hubo que operar «radiando a ciegas». Los servicios de combustible, cuando los había, eran activados mediante una pasada sobre el domicilio del encargado. El libro de registro de movimientos se encontraba en un cajón al borde de la pista. No obstante, se siguió volando noche y día.

Las condiciones de alojamiento y comidas fueron malas al comienzo pero mejoraron progresivamente, gracias a las excelentes relaciones con los órganos administrativos de la ONU y a la propia capacidad de adaptación del destacamento. En este sentido hay que destacar la enorme facilidad de re- 


\section{Pedro Bernal Gutiérrez}

lación del personal español con la población civil y el nivel de confianza depositada en el destacamento español por los dos bandos enfrentados ${ }^{29}$.

$\mathrm{El}$ aspecto sanitario no planteó grandes problemas, gracias a la larga experiencia acumulada en Guinea Ecuatorial, por el contrario, la capacidad disponible sirvió para proyectar la ayuda del destacamento español sobre otros contingentes, los ciudadanos españoles de la flota pesquera española en Namibia y sobre la población local.

Si desde el punto de vista operativo y logístico se pudo materializar con éxito el despliegue y operación continuada durante un año, gracias a la larga experiencia acumulada por el Ejército del Aire, no es menos cierto que la colaboración con los representantes diplomáticos y el apoyo recibido en los momentos más difíciles fueron elementos decisivos para el éxito de la misión

\section{Consideraciones finales}

Calificar al Ejército del Aire como pionero en misiones de paz y humanitarias no es simplemente hacer referencia a una acción particular alejada en el tiempo, más o menos homologable a ese tipo de misiones y en la que se dieran las circunstancias de primera vez, de incertidumbre e incluso de riesgo que le den la categoría de récord.

$\mathrm{Su}$ verdadero sentido es el de reflejar la vocación, el espíritu de servicio, y la capacidad para apoyar a la acción exterior de España y llevar a cabo actividades en favor de la convivencia humana, de la paz y de la solidaridad entre los pueblos, realizadas mucho antes de que esas actividades pudiesen ser definidas formalmente y ser integradas en las políticas, doctrinas y planes operativos de países u organizaciones internacionales.

La cristalización de esa predisposición en una capacidad material para emplear de forma organizada y flexible cualquiera de los medios del Ejército del Aire en el marco de las actuales Operaciones de Paz, se produjo a través de un proceso que se inició hace varias décadas, con el primer vuelo de ayuda, y marcó sucesivos hitos al afrontar en fechas tempranas nuevos desafíos que obligaron a superar niveles crecientes de dificultad, al mismo tiempo que las misiones de apoyo al Ejército de Tierra y la Armada también aumentaron progresivamente en la medida que las Fuerzas Armadas en su conjunto se fueron implicando cada vez más en este tipo de operaciones.

En nuestros días ese proceso culminó con la participación de nuestras unidades de combate en una operación compleja, junto a las fuerzas aéreas mejor equipadas y adiestradas de la OTAN, compartiendo con ellas precisamente su bautismo de fuego. 


\section{El Ejército del Aire pionero en operaciones de paz...}

\section{Notas}

1 Como hitos más destacables cabe citar la adhesión al Tratado del Atlántico Norte (OTAN) en 1981, la firma del Convenio de Cooperación para la Defensa con los Estados Unidos en 1988 y el ingreso en la Unión Europea Occidental también en 1988.

${ }^{2}$ Ministerio de Defensa: Libro Blanco de la Defensa 2000 - (Madrid), Capítulo III: La Política de Defensa española, pp.55-77.

${ }^{3}$ En el preámbulo de la DDN 1/00 se hace mención a la anterior Directiva 1/96 reconociendo que "se han alcanzado en gran medida los objetivos básicos de actuación" fijados en ella y «muy en particular en cuanto se refiere a la consolidación de la presencia internacional de España y al incremento de la eficacia y del grado de disponibilidad de las unidades militares".

4 La primera operación de mantenimiento de paz fue decidida por el Consejo de Seguridad en mayo de 1948, desde entonces se han realizado más de medio centenar.

5 España, desde su ingreso en la ONU en 1955, no participó en ninguna Operación de Mantenimiento de la Paz ni aportó medios económicos ni apoyo logístico en forma de "otras contribuciones" hasta 1988, aunque sí estuvo presente en el Comité especial de Operaciones de Mantenimiento de la Paz desde 1965, participando en sus reuniones y en los estudios y trabajos de seguimiento.

6 Una idea del aumento de las misiones de la ONU en esta segunda etapa, la da el hecho de que siendo su duración aproximadamente la cuarta parte de la anterior, el número de misiones iniciadas es casi cuatro veces mayor.

7 La Revista de Aeronáutica y Astronáutica publica en su número 704 Junio 2001 un dossier sobre la operación "Allied Force» en donde se describen y valoran los aspectos más interesantes de la participación del Ejército del Aire en la primera intervención armada de la OTAN.

8 Página Web del Ministerio de Defensa: participación española en misiones de paz. http://10.7.64.12/mde/mision/mision6.htm.

9 Del libro «Medio siglo de la Aviación en Getafe» de Manuel de la Peña Martín, pág. 150. Edición mayo de 1998. LERKO PRINT S.A.. Madrid.

10 Información obtenida en el Ala 35, extraída del documento "Resumen histórico" de la Unidad.

11 Información obtenida de la publicación del CECAF de 1998: XXV ANIVERSARIO DEL T.10 HERCULES. Cap.4. Ayuda Humanitaria y Cap.5. Apoyando el cumplimiento de las Resoluciones del Consejo de Seguridad de la ONU, pp.81-101. En los casos de discrepancia con los datos relacionados con el T-10 ofrecidos por otras fuentes, se le ha dado preferencia a esta publicación dedicada exclusivamente al Ala 31.

12 Información obtenida en el Ala 31, extraída del documento "Reseña histórica (I)» de la Unidad.

13 Revista de Aeronáutica y Astronáutica, número 468 del mes de diciembre de 1979, pág. 1156, «Misión Nicaragua 79».

14 Hasta febrero de 1987 la unidad se denominó 401 Escuadrón.

15 Información obtenida en el 45 Grupo, extraída de su curriculum de ayuda humanitaria, misiones de paz, cooperación internacional y apoyo a la población del 45 Grupo de Fuerzas Aéreas.

${ }^{16}$ Revista de Aeronáutica y Astronáutica, número 236 de julio de 1960. El SAR español en su V Aniversario. 
17 Recientemente, con ocasión de la operación ONUMOZ en Mozambique, iniciada en marzo de 200 participaron medios del 803 Escuadrón del SAR y de las FAMET del Ejército de Tierra.

18 En muchos de esos casos la coordinación de la obtención y distribución de la ayuda humanitaria era llevada a cabo por la Cruz Roja.

19 Ministerio de Defensa. MISIONES DE PAZ.MILITARES ESPAÑOLES EN EL MUNDO.1979-1991. Cap. III. Misiones en Africa. Guinea Ecuatorial. Pp.100-107.

20 En teletipo $\mathrm{n}^{\mathrm{a}} \mathbf{1 1 1 9 0 - D O P}$ de fecha 18.08.79, el Jefe del Estado Mayor del Aire ordenaba al General Jefe del MATRA el desplazamiento de dos aviones T-12 "Aviocar» a Malabo «para atender las peticiones de transporte de la Embajada de España y cuya duración se prevé bastante prolongada".

${ }^{21}$ Los sobrevuelos correspondían a Mauritania, Senegal, Gambia, Guinea Bissau, Guinea Conakry, Sierra Leona, Liberia, Costa de Marfil, Ghana, Togo, Benin, Nigeria, Camerún y Guinea Ecuatorial.

22 De acuerdo con datos del Ala 35 y lo publicado en Revista de Aeronáutica y Astronáutica/Enero - Eebrero de 1993. El Ala 35 finaliza su destacamento en Malabo, pág. 11, el Ala 35 realizó 6.116 salidas con 11.426 horas de vuelo y transportó 85.400 pasajeros y 1.623 toneladas de carga. Según los datos obtenidos en el Ala 37, esta Unidad realizó por su parte 1.039 salidas, 1.536 horas de vuelo y transportó 6.832 pasajeros y 91 toneladas.

${ }_{23}$ Ministerio de Defensa. MISIONES DE PAZ. MILITARES ESPAÑOLES EN EL MUNDO.1979-1991. Cap. III. Misiones en Africa. UNTAG, pp.79-95.

24 En 1966 la Asamblea General consideró oficialmente que había expirado el mandato concedido a Sudáfrica en 1920 para administrar a la antigua colonia alemana en el Sudoeste de Africa y creó el Consejo de UN para Namibia. Ese mismo año inició la guerra de guerrillas el SWAPO (South West Africa People's Organization).

25 Ministerio de Defensa. MISIONES DE PAZ. MILITARES ESPAÑOLES EN EL MUNDO.1979-1991. Cap. II. España, con los boinas azules, pp.40.

26 En los trabajos iniciales se manejó una cifra de 180 personas que posteriormente fue reducida a 150 y a ultima hora quedó en 85 , cuando también se redujo de 10 a 8 el número de aviones, al aplicar unos criterios operativos más realistas, en los que se conjugaban las necesidades de la misión con la capacidad del Ejército del Aire para sostener el destacamento durante un año, sin desatender sus compromisos habituales.

27 Además de pintar los aviones con los colores de la ONU, las condiciones de operación exigían un equipo de navegación, en este caso el equipo OMEGA, comunicaciones HF y un buen radar meteorológico. Además era preciso contar con un alto potencial de horas de vuelo, ya que no estaba previsto el relevo para inspecciones programadas. La preparación tuvo que hacerse en unas pocas semanas por lo que hubo que repartir las diferentes acciones entre las tres Maestranzas Aéreas.

$28 \mathrm{Al}$ comienzo de la misión se vivieron momentos de gran tensión con ocasión del asesinato del abogado del SWAPO, Luboski y de un atentado a instalaciones de la ONU. Más tarde, en dos ocasiones del mes de noviembre de 1989, se tuvieron noticias de aumento de la tensión en el norte, al tiempo que las comunicaciones telefónicas y por radio con los dos destacamentos, principalmente con el de Ondangwa, se vieron gravemente perturbadas. Las tripulaciones permanecieron listas durante 24 horas para evacuar de forma inmediata.

29 El Secretario General del SWAPO, Sam Nujoma, utilizaba el edificio del destacamento como zona de paso segura para acceder al aeropuerto de Eros. En alguna ocasión coincidió con la visita amistosa de algún representante sudafricano. Obviamente, se les mantuvo separados e ignorantes el uno del otro. 